\title{
Survival outcomes of unilateral retinoblastoma based on pathological risk stratification-experience at a tertiary care centre in Pakistan
}

\author{
Najma Shaheen ${ }^{1}$, Naila Inayat ${ }^{1}$, Sehar Bashir ${ }^{2}$, Umer Nisar Sheikh², Muhammad Abu Bakar ${ }^{3}$ and Palwasha Rehman ${ }^{1 a}$ \\ ${ }^{1}$ Department of Pediatric Oncology, Shaukat Khanum Memorial Cancer Hospital and Research Centre, 7A, Johar Town, Lahore 54782, Pakistan \\ ${ }^{2}$ Department of Pathology, Shaukat Khanum Memorial Cancer Hospital and Research Centre, 7A, Johar Town, Lahore 54782, Pakistan \\ ${ }^{3}$ Epidemiologist and Bio-statistician-Cancer Registry, Shaukat Khanum Memorial Cancer Hospital and Research Centre, 7A, Johar Town, Lahore 54782, \\ Pakistan \\ ahttps://orcid.org/0000-0002-3745-380X
}

\begin{abstract}
Retinoblastoma (RB) is the most common childhood intraocular malignancy. In highincome countries over the past decade, upfront enucleation for unilateral RB is least favoured due to other alternatives that can help in globe preservation, but in low-middle income countries it is still the preferred option due to lack of resources and expertise. The treatment of RB after enucleation is tailored based on the histopathological risk features, as adjuvant chemotherapy with high-risk features reduces the risk of metastasis. The aim of our study was to analyse the survival outcomes of adjuvant therapy based on histopathological risk stratification in patients who underwent upfront enucleation for unilateral RB with advanced disease. A retrospective study was carried out at Shaukat Khanum Memorial Cancer Hospital and Research Centre, Pakistan. A total 113 patients (aged 3 months till 16 years) diagnosed with unilateral RB who had upfront enucleation from July 2009 till January 2019 were included in this study. The mean age of diagnosis was 37.4 months $( \pm 24.5)$ and male-to-female ratio of 1.3:1. The most common clinical presentation was leukocoria (74.3\%). Patients who underwent enucleation had advanced disease; group D present in $62.8 \%$ followed by group E (32.7\%). Histopathology revealed high-risk features in 29 patients (25.7\%) and intermediate risk in 54 patients (47.8\%). Disease progression and relapse was seen in patients with high-risk histopathological features. The 4-year over-all survival and EFS observed for this cohort was $74 \%$ and $71 \%$. Awareness about the early symptoms among the general population and health care personnel at a nationwide level is needed to facilitate early detection and lessen disease related morbidity and mortality.
\end{abstract}

Keywords: children, enucleation, retinoblastoma, pathology

\section{Introduction}

Retinoblastoma (RB) is the most common intraocular malignancy in childhood and accounts for about $2 \%$ of all childhood cancers [1]. The incidence of RB ranges from 1 case per 15,000-20,000 live births worldwide [2]. RB occurs due to germline or somatic
Correspondence to: Palwasha Rehman Email: RehmanPalwasha@outlook.com

ecancer 2022, 16:1360

https://doi.org/10.3332/ecancer.2022.1360

Published: 01/03/2022

Received: 26/08/2021

Publication costs for this article were supported by ecancer (UK Charity number 1176307).

Copyright: $($ ) the authors; licensee ecancermedicalscience. This is an Open Access article distributed under the terms of the Creative Commons Attribution License (http:// creativecommons.org/licenses/by/4.0), which permits unrestricted use, distribution, and reproduction in any medium, provided the original work is properly cited. 
mutations. Loss of function of RB 1 gene on chromosome 13q results in the germline (hereditary) RB, which commonly presents as bilateral eye involvement in early age group. Somatic RB (non-heritable RB) results from loss of function of RB1 gene in the retina; patients will usually have unilateral, unifocal disease and present at a later age compared to the heritable type [3].

In high-income countries, due to early detection and optimal health facilities, the overall survival (OS) of RB has increased to more than 95\%; hence, the aim of treatment has been shifted to globe salvage [4]. In low-middle income countries (LMICs), low-socioeconomic status, advance disease and abandonment are the main risk factors leading to poor outcomes among RB patients [5, 6]. Asia-pacific region contrib-utes significantly to RB; almost $43 \%$ of the children diagnosed globally belong to this region. India ranking in the first place where 1,500 cases are detected annually, whereas Pakistan stands at the fourth place [7]. The annual crude incidence of RB in children under the age of 5 and 10 years in Karachi (Pakistan) was reported to be 4.0/100,000 and 2.4/100,000 [8].

Majority of the intraocular disease (small or medium sized) can be treated with globe preserving strategy, however primary enucleation remains the treatment of choice for advanced disease [9]. RB can be stratified based on clinical presentation and histopathological features. Clinical features such as irreversible neovascular glaucoma, aseptic orbital cellulitis (group E disease) is associated with high-risk histopatho-logical features and require adjuvant chemotherapy after enucleation as there is always an increased risk of metastasis $[10,11]$.

In high-income countries, the incidence of histopathological high-risk features after enucleation had declined over the years due to early diagnosis and referral; however, cases are still reported from LMICs like Pakistan [12, 13]. Post enucleation histopathological features play an important role in assessing the disease severity, results from high-income countries cannot be generalized as presentation of disease differs in LMICs [14]. The purpose of our study was to analyse the survival outcomes of adjuvant therapy based on histopathological risk stratifica-tion in patients who underwent upfront enucleation for unilateral RB with advanced disease.

\section{Materials and methods}

\section{Patients and diagnostic evaluation}

Shaukat Khanum Memorial Cancer Hospital and Research Centre is among one of the leading cancer care charity hospitals of the country, where oncology patients (adults/children) receive treatment irrespective of their ability to pay. It is 195 beds (in-patient) facility and approxi-mately 500-550 paediatric oncology patients are registered annually.

Hospital-based electronic data of children with RB from July 2009 to January 2019 was reviewed after approval from the institutional review board. A total of 113 children with unilateral RB were identified who had upfront enucleation and were naive for chemotherapy.

RB diagnosis was made either by indirect ophthalmoscopic examination under anaesthesia (EUA) or on histopathology confirmation after enucleation [15]. Extent of disease evaluation included ocular EUA, B scan (where applicable), magnetic resonance imaging scan or contrast enhanced computerised tomography scan of brain and orbit in all patients. Cerebrospinal fluid examination with cytospin, bone marrow biopsy and aspiration with immunocytology were done for disease staging [16]. According to International Retinoblastoma Classification (IIRC), the intraocular disease was grouped [17]. All cases were subjected to histological examination for tumour grade/differentiation and evaluation of high-risk features, which included the presence and level of optic nerve involvement, post-laminar and extension to the optic nerve resection margin, location and extent of invasion of uveal tract. Invasion was measured in millimetres and labelled as massive if more than $3 \mathrm{~mm}$ in diameter. Other histological features such as necrosis, calcification, involvement of other ocular structures; anterior chamber, ciliary body sclera was also noted [18].

\section{Treatment plan and follow-up}

Adjuvant chemotherapy was offered based on histological features, patients with low-risk features (prelaminar invasion, focal choroidal inva-sion ( $<3 \mathrm{~mm}$ ) and ciliary body involvement) were offered no chemotherapy. Those with intermediate-risk features (massive choroidal invasion with $>3 \mathrm{~mm}$, and anterior chamber invasion) were offered four courses of chemotherapy and patients with high-risk features (optic nerve cut 
end and scleral invasion) were given six courses of chemotherapy [19]. The chemotherapy protocol (vincristine $1.5 \mathrm{mg} / \mathrm{m}^{2}$, carboplatin 600 $\mathrm{mg} / \mathrm{m}^{2}$ and etoposide $300 \mathrm{mg} / \mathrm{m}^{2}$ ) was given 21 days apart with the neutrophil count $\geq 1 \times 10^{9} / \mathrm{L}$ and platelets $\geq 100 \times 10^{9} / \mathrm{L}$. External Beam Radiotherapy at a dose of $40 \mathrm{~Gy}$ in 20 fractions was offered to patients with optic nerve cut end invasion [20]. Post treatment patients had 3 monthly follow-up for 2 years and then after every 6 months till the age of 10 years.

\section{Statistical analysis}

Statistical analysis was performed using the Statistical Package for the Social Sciences version 20. Mean and standard deviation were reported for quantitative data like age and frequency and percentages were reported for qualitative data like; sex, presenting signs and IIRC Group. OS and event free survival (EFS) was calculated using Kaplan-Meir curve method. The EFS was calculated from the date of diagnosis until date of death or date of progression of disease or date of relapse. OS was calculated from date of diagnosis till date of death. Log rank was used to calculate the survival difference and $p$-value of $\leq 0.05$ was considered to be statistically significant.

\section{Result}

\section{Patient characteristics}

A total 113 patients were included in this report. The mean age of diagnosis was $37.4 \pm 24.5$ months, with male-to-female ratio of $1.3: 1$. The median follow up time was 34.9 months (SD \pm 27.8) (Interquartile range (IQR)- 10-62). The eldest patient in our group was 15 years old. Eighty-two patients (72.6\%) belonged to urban areas, whereas 6.2\% patients were referred from neighbouring country (Afghanistan).

The most common clinical presentation of disease was leukocoria (74.3\%), and the most common clinical group seen was group $\mathrm{D}$ (60.2\%) (Table 1) in this report. Seventy-two patients (63.7\%) had upfront enucleation and one child had exenteration from outside facility. Forty patients (35.4\%) underwent enucleation at our institute. The mean number of days from the enucleation to start of chemotherapy was 25.3 \pm 26.4 days (range $0-130$ days).

\section{Outcomes}

In our cohort, advance disease on presentation (group D and E) had intermediate and high-risk features on histopathology requiring adjuvant chemotherapy with $p$ value of 0.02 .

Twenty-six enucleated (23\%) eyes had well differentiated tumour histology, 31 eyes (27.4\%) had moderately differentiated histology, whereas 53 eyes (46.9\%) had poorly differentiated histology. Thirty patients (26.5\%) were stratified as low risk based on histopathological features of enucleated eye, 54 patients (47.8\%) as intermediate risk, whereas 29 patients (25.7\%) had high-risk features. Intermediate and high-risk patients were offered chemotherapy, whereas radiation therapy was only offered to patients stratified as high risk.

The low-risk group $(n=30)$ had good outcomes; relapse disease was seen in one patient only. Disease progression and relapse were seen in the groups having intermediate and high-risk features on histopathology. Among the intermediate risk group, one patient and three patients with high-risk histopathological features died during treatment due to sepsis (Table 2).

At the end of treatment, complete remission was achieved in 83 patients (73.5\%). Death as first event was observed in four patients (3.5\%). Disease progression was documented in seven patients (6.2\%), among them three patients refused further treatment, whereas four patients died due to disease burden. Disease relapse was reported in 12 patients (10.6\%). The average time of relapse noted after the end of treatment was 10 months (range 2-36 months). Seven patients had intracranial relapse; palliative radiation therapy was given to them. Leptomeningeal metastasis was seen in three patients. One patient had pineal blastoma along with spinal metastasis 18 months after end of therapy, whereas one patient had extraocular relapse. Unfortunately, seven patients (5.3\%) abandoned therapy during their course of treatment. 
Table 1. Patient and tumour characteristics $(n=113)$.

\begin{tabular}{|l|l|}
\hline Patient characteristics & $n$ (Percentages \%) \\
\hline Mean age at presentation & $37.4 \pm 24.5$ months \\
\hline Gender & \\
\hline Male & $65(57.5 \%)$ \\
\hline Female & $48(42.5 \%)$ \\
\hline Presenting signs ${ }^{\text {a }}$ & \\
\hline Leukocoria & $84(74.3 \%)$ \\
\hline Proptosis & $11(9.7 \%)$ \\
\hline Red eye & $6(5.3 \%)$ \\
\hline Vision loss & $7(6.2 \%)$ \\
\hline Squint & $3(2.7 \%)$ \\
\hline Ptosis & $1(0.9 \%)$ \\
\hline IIRC Group & \\
\hline Group C & $5(4.4 \%)$ \\
\hline Group D & $68(60.2 \%)$ \\
\hline Group E & $40(35.4 \%)$ \\
\hline Pathology & \\
\hline Anterior segment invasion & $38(33.6 \%)$ \\
\hline Ciliary body invasion & $16(14.1 \%)$ \\
\hline Massive choroidal invasion & $20(17.7 \%)$ \\
\hline Scleral invasion & $12(10.6 \%)$ \\
\hline Pre-laminar invasion & $15(13.3 \%)$ \\
\hline Post-Laminar invasion & $33(29.2 \%)$ \\
\hline Optic nerve cut end invasion & $29(25.7 \%)$ \\
\hline 4-year OS & $74 \%$ \\
\hline 4-year EFS & $71 \%$ \\
\hline One patient had strong family history, had upfront \\
\hline
\end{tabular}

Table 2. Outcomes of disease regarding histopathological risk stratification.

\begin{tabular}{|l|c|c|c|c|c|c|}
\hline & \multicolumn{5}{|c|}{ Outcomes } & \multicolumn{2}{|c|}{$\begin{array}{c}\text { Disease } \\
\text { Risk group based on } \\
\text { histopathological features }(\boldsymbol{n}=113)\end{array}$} & $\begin{array}{l}\text { Complete } \\
\text { response }\end{array}$ & $\begin{array}{c}\text { Disease } \\
\text { relapse }\end{array}$ & $\begin{array}{c}\text { Death } \\
\text { progrendonment }\end{array}$ & $p$ value \\
\hline Low risk & 29 & 1 & 0 & 0 & 0 & $<0.05$ \\
\hline Intermediate risk & 43 & 6 & 1 & 1 & 3 & \\
\hline High risk & 11 & 5 & 6 & 3 & 4 & \\
\hline
\end{tabular}



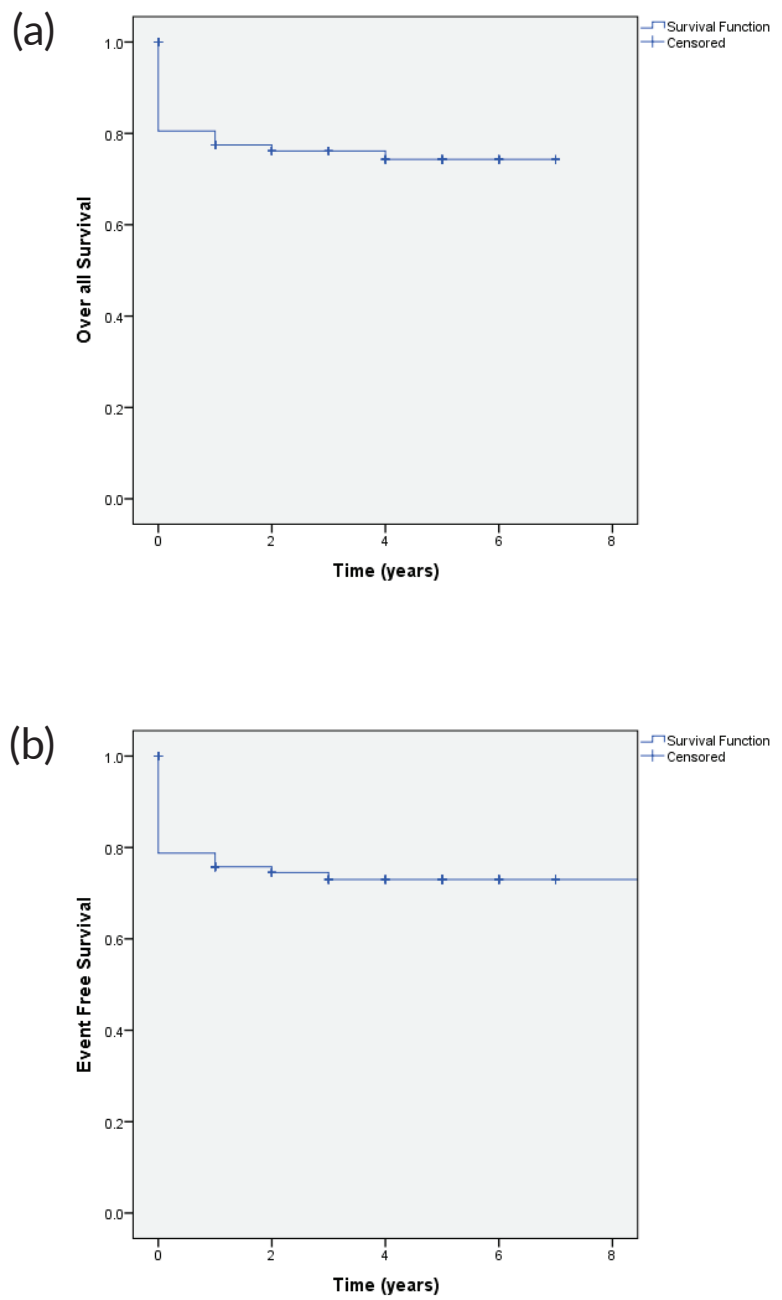

Figure 1. (a): OS and (b): EFS.

The 4-year OS and EFS observed for this cohort was $74 \%$ and $71 \%$, respectively (Figure 1). OS of patients with group C at 4 years was $80 \%$, group D $82.4 \%$, and group E was $65 \%$, respectively. The event-free survival in group C, D and E was $80 \%$, $79 \%$, and $59 \%$, respectively (Figure 2).

The 4-year OS survival based on histopathological risk stratification in low-risk group was $96.7 \%$, in intermediate risk group $79.6 \%$ and $43 \%$ in high-risk group, whereas 4-year EFS in low-risk group was $96 \%, 76 \%$ in intermediate risk and $41 \%$ in high-risk group respectively (Figure 3).

\section{Discussion}

Asia-pacific region of the world contributes significantly to the diagnosis of RB, Pakistan is among the top ten countries of the regions that shares significant burden of this disease [21]. The clinical presentation of the RB is variable including leukocoria, strabismus, vision loss or painful eye [22]. Leukocoria was the most common clinical presentation (74.3\%) seen in our patients followed by proptosis, comparable to 
other LMICs [23]. Along with leukocoria, proptosis and vision loss were the usual presenting clinical signs associated with advanced group disease $D$ and $E$ ( $p$ value 0.002 ). The mean age of presentation among our cohort was 37.4 months, which is higher than our neighbouring countries where it was reported to be 20 months [24]. This may reflect delay in diagnosis and referral of our study population to the cancer treatment centre. A male predominance was seen in our patients, other studies from Asia have reported similar results, although no sex predilection has been seen in RB and the differences mainly can be due to gender discrimination $[25,26]$.

The advanced group $\mathrm{D}$ and $\mathrm{E}$ disease among our patients had high-risk histopathological features and required adjuvant chemotherapy. Optic nerve cut end invasion termed as high-risk feature was present in $25.7 \%$ of patients and they received radiation therapy along with adjuvant chemotherapy. Globe salvage is being promoted for group D eyes with the introduction of the new treatment modalities like intra-arterial chemotherapy (IAC), but disease relapse can still not be ignored. However, for group E disease primary enucleation is still the treatment of choice [27]. Unfortunately, in our setting we lack the expertise of administration of IAC.

\section{(a)}

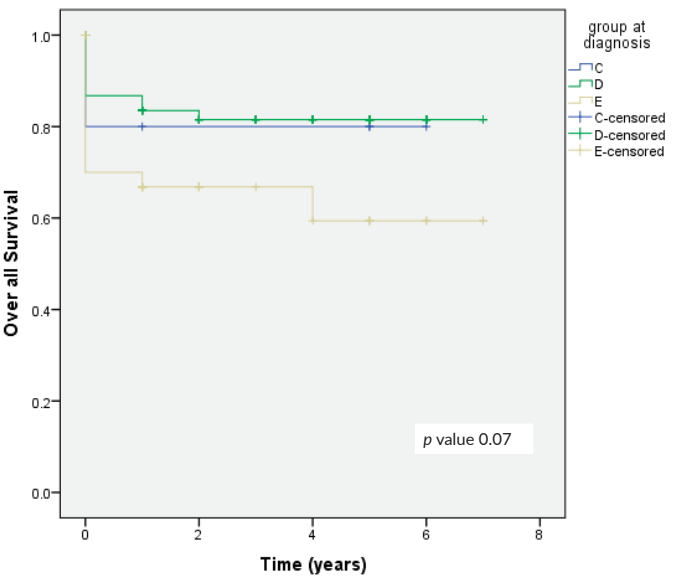

(b)

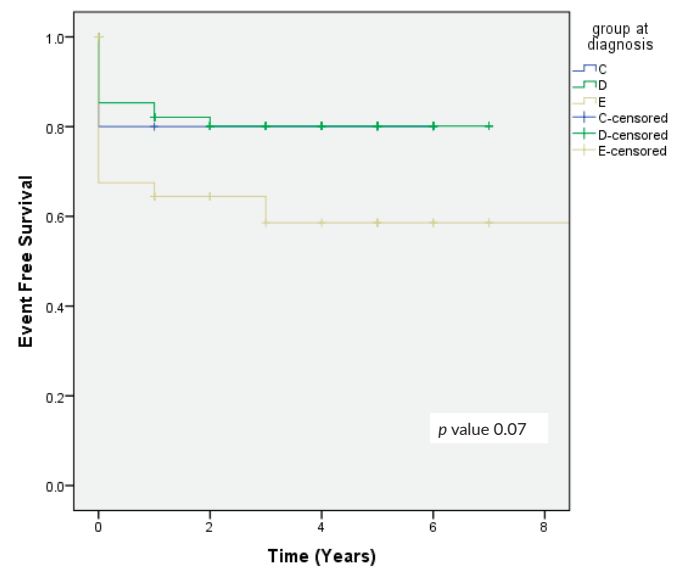

Figure 2. (a): OS in relation to clinical group and (b): EFS in relation to clinical group. 

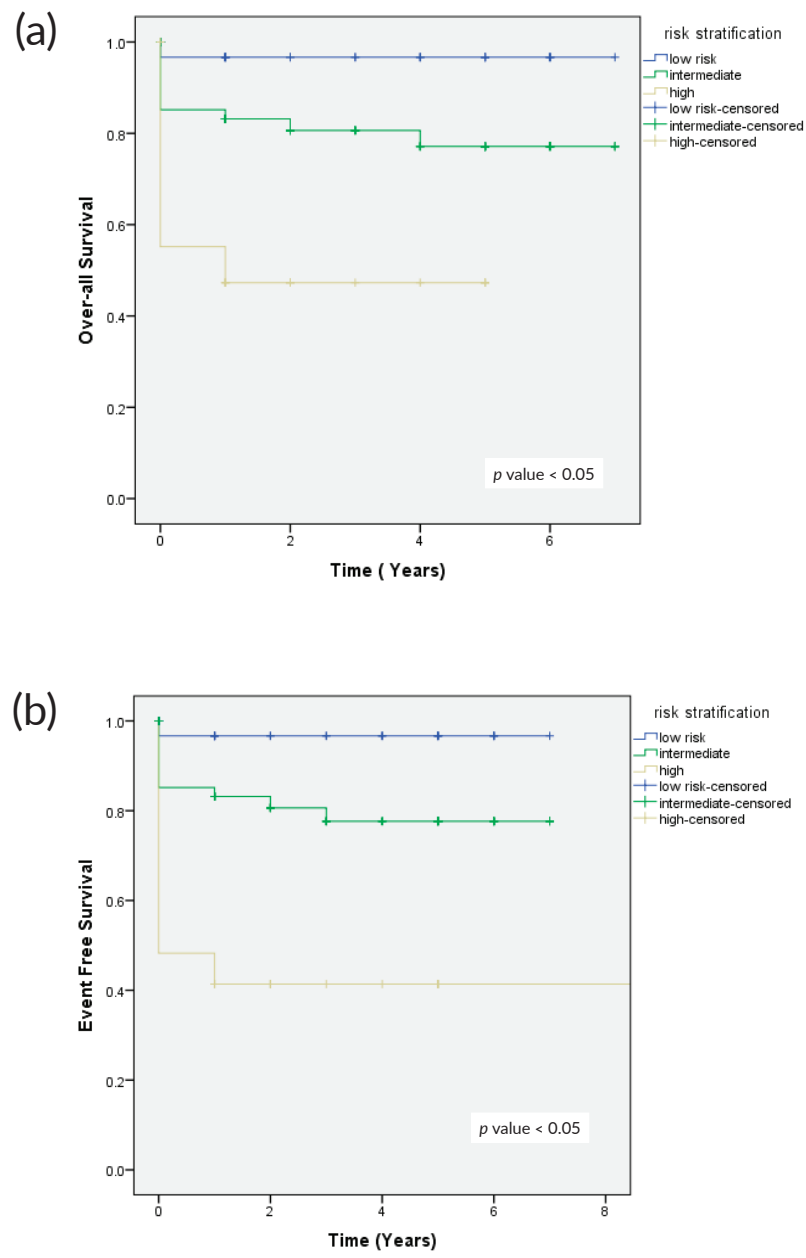

Figure 3. (a): OS as per risk stratification and (b): EFS as per risk stratification.

Previous study has shown that timely enucleation followed by adjuvant chemotherapy in high-risk histopathology reduces the risk of metastatic disease [28]. In our data, 15 patients had disease progression and metastasis in intermediate and high-risk group who received adjuvant chemotherapy after 2 weeks of enucleation. When we analysed the outcomes based on initiation of adjuvant chemotherapy, the patients who received chemotherapy within 2 weeks of enucleation had better survival compared to those who received after 2 weeks ( $94.1 \%$ versus 64.1\%, $p$-value 0.02) (Figure 4).

The histopathological feature of RB plays major role in predicting outcome of the disease [29]. On microscopic level, RB reveals small hyperchromatic cells with high nuclear to cytoplasmic ratio, the tumour is differentiated into well differentiated to poorly differentiated (based on the Homer-Wright rosettes) [30]. In our data, 62 patients (54.8\%) with poorly and moderately differentiated histology had high risk features and were given adjuvant chemotherapy. Disease relapse and progression was also seen among these patients, although statistically it was not significant ( $p$ value- 0.26). Kashyap et al [31] reported that poorly differentiated tumours are associated with multiple high-risk features, as compared to well differentiated tumours ( $p$ value of $<0.001$ ). 


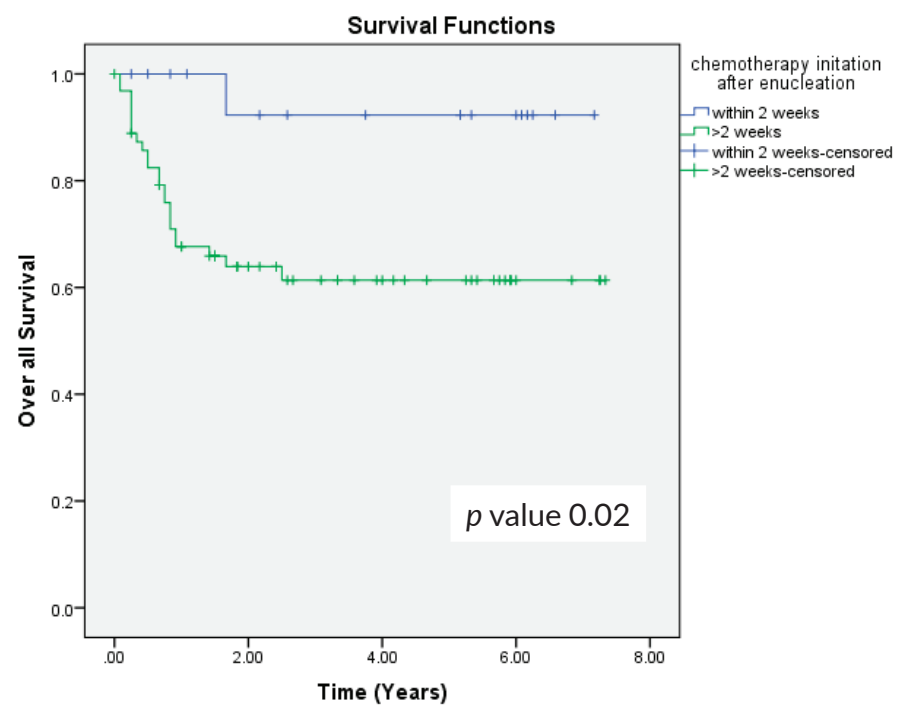

Figure 4. OS in relation to the initiation of chemotherapy after enucleation.

Early diagnosis and treatment can improve outcomes in RB. In our cohort, disease progression was seen in $6.2 \%$ patients while on treatment, which is within the estimated range for LMICs [32]. Slightly better outcomes were seen in patients who had enucleation at our facility as compared to those who had at outside facility (EFS 75.3\% versus 70\%).

Five patients (4.4\%) in our study had group C disease on EUA, but they underwent upfront enucleation (at an outside facility). Majority of our patients $(72.6 \%)$ that presented with advanced disease belonged to urban areas. Due to lack of expertise and limited resources in our country, novel therapies for globe salvage could not be offered, it can be speculated that the treatment option of upfront enucleation can be one of the risk factors that lead to advanced disease presentation. Since it is retrospective study exact risk factors that lead to advance disease could not be assessed.

In our report, seven patients (5.3\%) abandoned treatment, even though free of cost therapy was offered at our centre. Abandonment had always been a challenge when treating oncology patients, high rate of abandonment has been seen in RB patients in LMICs, intensive chemotherapy regimens and mutilating surgical procedures such as enucleations and prolong follow up can be considered as a risk factor [33]. In the central American Association of Pediatric Hematology Oncology II trial, 102 patients $(n=161)$ received upfront enucleation and 59 patients underwent delayed enucleation. Although they were successful in reducing the abandonment in patients with advanced disease, the children with upfront enucleation had better outcomes as compared to those with delayed enucleation (5-year OS $94 \%$ versus $74 \%$ : $p$ value $<0.001)$ [34].

This study is retrospective with some inherited limitations, as data was collected through online electronic media and majority of our patients were referred after enucleation, the exact onset of symptoms could not be determined. Secondly, to determine the intraocular disease group in children, we relied on the examination done by referring ophthalmologist. In resource limited setting, patients with advanced unilateral disease (group D or E) without treatment modalities for globe salvage upfront enucleation can be considered. RB awareness programs for early recognition and referrals, multiple disciplinary team (MDT) meetings with experts or telemedicine clinics are the ways through which management of RB could be implemented in LMICs, resulting in early recognition and reducing mortality rates [35]. We have started similar program as National RB MDT under guidance of Pakistan Society of Pediatric Oncology (PSPO) where difficult and complex cases are being discussed. Recently, for uniformity of staging and treatment we have proposed nationwide protocol of RB under leadership of PSPO which is to be adopted by all oncology centres and ophthalmologists of the country, the results of which are yet to be determined. 


\section{Conclusion}

Risk stratification based on histopathological features of the enucleated eye is an effective way of tailoring adjuvant therapy. Significant number of patients avoided chemotherapy with very low risk of relapse. Globe salvage in advanced disease is possible with the introduction of newest modalities like IAC, but it has limited availability due to cost effectiveness and lack of expertise in resource limited setting. Hence, in advanced unilateral RBs, we recommend upfront enucleation for risk stratification and treatment accordingly in our setting.

\section{Conflicts of interest}

The authors declare no conflict of interest.

\section{Disclosure}

The abstract was presented as an e-poster at SIOP 2021.

\section{Funding}

None.

\section{References}

1. Siegel RL, Miller KD, and Jemal A (2019) Cancer statistics, 2019 CA Cancer J Clin 69(1) 7-34 https://doi.org/10.3322/caac.21551 PMID: 30620402

2. Stacey AW, Bowman R, and Foster A, et al (2021) Incidence of retinoblastoma has increased: results from 40 European countries Ophthalmology 128(9) 1369-71 https://doi.org/10.1016/j.ophtha.2021.01.024 PMID: 33508429

3. Dimaras H and Corson TW (2019) Retinoblastoma, the visible CNS tumor: a review J Neurosci Res 97(1) 29-44 https://doi.org/10.1002/ $\underline{\text { jnr.24213 }}$

4. Chen Q, Zhang B, and Dong Y, et al (2018) Comparison between intravenous chemotherapy and intra-arterial chemotherapy for retinoblastoma: a meta-analysis BMC Cancer 18(1) 1-1 https://doi.org/10.1186/s12885-018-4406-6

5. Goolam S, Kana H, and Welsh N, et al (2018) A 20-year retrospective review of retinoblastoma at two tertiary academic hospitals in johannesburg, South Africa Ocular Oncol Pathol 4(3) 170-175 https://doi.org/10.1159/000481508

6. Essuman V, Ntim-Amponsah CT, and Akafo S, et al (2010) Presentation of retinoblastoma at a paediatric eye clinic in Ghana Ghana Med J 44(1)

7. Jain M, Rojanaporn D, and Chawla B, et al (2019) Retinoblastoma in Asia Eye 33(1) 87-96 https://doi.org/10.1038/s41433-018-0244-7 PMCID: 6328585

8. Bhurgri Y, Muzaffar S, and Ahmed R, et al (2004) Retinoblastoma in Karachi, Pakistan Asian Pac J Cancer Prevent 5(2) 159-163

9. Ancona-Lezama D, Dalvin LA, and Shields CL (2020) Modern treatment of retinoblastoma: a 2020 review Indian J Ophthalmol 68(11) 2356 https://doi.org/10.4103/ijo.IJO_721_20 PMID: 33120616 PMCID: 7774148 
10. Hudson LE, Mendoza P, and Hudson WH, et al (2018) Distinct gene expression profiles define anaplastic grade in retinoblastoma Am J Pathol 188(10) 2328-2338 https://doi.org/10.1016/j.ajpath.2018.06.013 PMID: 30036517 PMCID: 6168968

11. Kaliki S, Shields CL, and Rojanaporn D, et al (2013) High-risk retinoblastoma based on international classification of retinoblastoma: analysis of 519 enucleated eyes Ophthalmology 120(5) 997-1003 https://doi.org/10.1016/j.ophtha.2012.10.044 PMID: 23399379

12. Kaliki S, Shields CL, and Eagle Jr RC, et al (2018) High-risk intraocular retinoblastoma: Comparison between Asian Indians and Americans from two major referral centers Retina 38(10) 2023-2029 https://doi.org/10.1097/IAE.0000000000001816

13. Yaqoob N, Mansoor S, and Aftab K, et al (2022) High risk histopathological factors in retinoblastoma in upfront enucleated eyes: an experience from a tertiary care centre of Pakistan Pak J Med Sci 38(ICON-2022)

14. Yaqoob N, Mansoor S, and Zia N, et al (2022) Chemotherapy induced histopathological changes in retinoblastoma, assessment of highrisk predictive factors \& its correlation with comorbid conditions Pak J Med Sci 38(ICON-2022)

15. Chantada GL, Gonzalez A, and Fandino A, et al (2009) Some clinical findings at presentation can predict high-risk pathology features in unilateral retinoblastoma J Pediatr Hematol/Oncol 31(5) 325-329 https://doi.org/10.1097/MPH.0b013e3181923cc5

16. Chantada G, Doz F, and Antoneli CB, et al (2006) A proposal for an international retinoblastoma staging system Pediatr Blood Cancer 47(6) 801-805 https://doi.org/10.1002/pbc.20606

17. Shields CL, Mashayekhi A, and Au AK, et al (2006) The International Classification of Retinoblastoma predicts chemoreduction success Ophthalmology 113(12) 2276-2280 https://doi.org/10.1016/j.ophtha.2006.06.018 PMID: 16996605

18. Eagle Jr RC (2009) High-risk features and tumor differentiation in retinoblastoma: a retrospective histopathologic study Arch Pathol Lab Med 133(8) 1203-1209 https://doi.org/10.5858/133.8.1203

19. Honavar SG, Singh AD, and Shields CL, et al (2002) Postenucleation adjuvant therapy in high-risk retinoblastoma Arch Ophthalmol 120(7) 923-931 https://doi.org/10.1001/archopht.120.7.923 PMID: 12096963

20. UKCCSG Retinoblastoma (2005) Working Group Guidelines for the Management of Children with Advanced Retinoblastoma following Primary Rnucleation (Version 1.0)

21. Usmanov RH and Kivelä T (2014) Predicted trends in the incidence of retinoblastoma in the Asia-Pacific region Asia-Pac J Ophthalmol 3(3) 151-157 https://doi.org/10.1097/APO.0000000000000060

22. Zia N, Hamid A, and Iftikhar S, et al (2020) Retinoblastoma presentation and survival: a four-year analysis from a tertiary care hospital Pak J Med Sci 36(1) S61 PMID: 31933609 PMCID: 6943119

23. Navo E, Teplisky D, and Albero R, et al (2012) Clinical presentation of retinoblastoma in a middle-income country J Pediatr Hematol Oncol 34(3) e97-e101 https://doi.org/10.1097/MPH.0b013e31821d18f9

24. Faranoush M, Mehrvar N, and Tashvighi M, et al Retinoblastoma presentation, treatment and outcome in a large referral centre in Tehran: a 10-year retrospective analysis Eye 35(2) 575-583 PMID: 32367000 PMCID: 8027402

25. Kaliki S, Patel A, and Iram S, et al (2019) Retinoblastoma in India: clinical presentation and outcome in 1,457 patients (2,074 eyes) Retina 39(2) 379-391 https://doi.org/10.1097/IAE.0000000000001962

26. Fabian ID, Khetan V, and Stacey AW, et al (2021) Sex, gender, and retinoblastoma: analysis of 4351 patients from 153 countries Eye 1-7

27. Ravindran K, Dalvin LA, and Pulido JS, et al (2019) Intra-arterial chemotherapy for retinoblastoma: an updated systematic review and meta-analysis J Neuroint Surg 11(12) 1266-1272 https://doi.org/10.1136/neurintsurg-2019-014909

28. Zhao J, Feng ZX, and Wei M, et al (2020) Impact of systemic chemotherapy and delayed enucleation on survival of children with advanced intraocular retinoblastoma Ophthalmol Retina 4(6) 630-639 https://doi.org/10.1016/j.oret.2020.02.015 PMID: 32387053 
29. Mendoza PR, Specht CS, and Hubbard GB, et al (2015) Histopathologic grading of anaplasia in retinoblastoma Am J Ophthalmol 159(4) 764-776 https://doi.org/10.1016/j.ajo.2014.12.014 PMCID: 4361305

30. Singh L and Kashyap S (2018) Update on pathology of retinoblastoma Int J Ophthalmol 11(12) 2011 PMID: 30588438 PMCID: 6288520

31. Kashyap S, Sethi S, and Meel R, et al (2012) A histopathologic analysis of eyes primarily enucleated for advanced intraocular retinoblastoma from a developing country Arch Pathol Lab Med 136(2) 190-193 https://doi.org/10.5858/arpa.2010-0759-OA PMID: 22288967

32. Canturk S, Qaddoumi I, and Khetan V, et al (2010) Survival of retinoblastoma in less-developed countries impact of socioeconomic and health-related indicators Brit J Ophthalmol 94(11) 1432-1436 https://doi.org/10.1136/bjo.2009.168062

33. Siddiqui DE, Ashraf MS, and Iftikhar S, et al (2018) Predictors of treatment abandonment for patients with pediatric cancer at Indus Children Cancer Hospital, Karachi, Pakistan Pediatr Blood Cancer 65(2) e26818 https://doi.org/10.1002/pbc.26818

34. Luna-Fineman S, Chantada G, and Alejos A, et al (2019) Delayed enucleation with neoadjuvant chemotherapy in advanced intraocular unilateral retinoblastoma: AHOPCA II, a prospective, multi-institutional protocol in Central America J Clin Oncol 37(31) 2875 https:// doi.org/10.1200/JCO.18.00141 PMID: 31536438 PMCID: 6823891

35. Singh G and Daniels AB (2016) Disparities in retinoblastoma presentation, treatment, and outcomes in developed and less-developed countries Semin Ophthalmol 31(4) 310-316 https://doi.org/10.3109/08820538.2016.1154177 PMID: 27127937 